Editorial

\title{
Forests: An International and Interdisciplinary Scientific Open Access Journal
}

\author{
Eric J. Jokela \\ Founding Editor-in-Chief of Forests, School of Forest Resources and Conservation, \\ University of Florida, P.O. Box 110410, 118 Newins-Ziegler Hall, Gainesville, FL 32611-0410, USA; \\ E-Mail: ejokela@ufl.edu; Tel.: +1-352-846-0890; Fax: +1-352-846-1277
}

Received: 16 January 2014 / Accepted: 17 January 2014 / Published: 21 January 2014

Forests was established to provide comprehensive coverage on the ecology, conservation and management of forests, with the first issue published in March 2010. As an international and multi-disciplinary journal, Forests has provided a forum for publishing process-based and applied scholarly articles that span the technological, environmental, cultural, economic, and social realm associated with the management, use, conservation, and understanding of forested ecosystems. By all accounts, Forests is well poised toward becoming a premier publication outlet in this diverse field of study. In its short tenure, Forests received its first Impact Factor in 2013 (1.094-Science Citation Index Expanded (SCIE)/Web of Science), which placed it 25th out of 62 Forestry journals. Notably, Forests ranked first among the open access journals in this category.

A unique aspect of Forests that has contributed to its early success relative to other forestry journals is its on-line and Open Access format. This provides free article access via the internet to scientists, practitioners, policy makers, students, and other interested persons who would otherwise not have access to subscribed forestry journals. The full text of published articles can be queried and retrieved from all search engines. As a result, the number of citations and article submissions to Forests has continued to rise each year. In 2013, 66 articles were published and they originated from 25 different countries. Authors from the USA, Canada, Germany, Sweden and Brazil accounted for the greatest number of published articles. In addition, the number of full text downloads of articles published in 2013 was 22,860 . The average publication time from first submission to publication was about 79 days in 2013, which was slightly higher than 71 days in 2012. It should be noted that beginning in January 2014, Forests will move from a quarterly to a monthly journal, with articles being released to the Web of Science in the same month that they were published. Article submissions are welcomed from a wide spectrum of subject areas, including: 
- forest ecology, management, and restoration

- forest economics, natural resource policy and planning

- $\quad$ silvicultural systems

- forest entomology, forest pathology

- forest ecophysiology and biology

- forest genetics, tree breeding and biotechnology

- climate change impacts, adaptation and mitigation in forests

- forest biomass, bioenergy, and carbon sequestration

- forest engineering

- tropical forests and management

- forest inventory, quantitative methods, and remote sensing

- forest soil management

- forest simulation modeling

- forest and nature based recreation

- $\quad$ wildland fire science and management

- wood properties

- human dimensions

- urban forests

Also contributing to the international recognition of Forests has been the timely publication of Special Issues that have focused on scientifically relevant and contemporary topics. In 2013, three special issues were published (Forest Restoration and Regeneration; Fusiform Rust Disease-Biology and Management of Resistance; Improving Wood Quality from Planted Forests) and 11 additional special issues are planned on a variety of topics, including papers from technical sessions held at the 24th IUFRO World Congress in Salt Lake City, Utah (USA).

As Editor-in-Chief, I am very appreciative of the high quality Editorial Board of Forests. Our diverse and eminent, 40-member scientific Editorial Board represents the breadth of forestry sub-disciplines and topical areas. Their combined and collective experience has ensured the highest degree of scientific rigor and review of all published articles. In addition, I would also like to recognize the valuable contributions made by manuscript reviewers (listed in Appendix), as this dedicated service represents an unselfish donation of their time and energy. As we all recognize the peer review process lends both scientific credibility and respectability to the journal. I have been very pleased with the conscientious and thorough reviews, suggestions, and comments provided by the Editorial Board Members and reviewers, which has clearly aided in improving manuscript quality.

I would also like to extend a special note of gratitude to the editorial office of Forests. Dr. Echo Zhang (Managing Editor), Dr. Martyn Rittman (Production Editor) and Dr. Shu-Kun Lin (Publisher) have done an excellent job. We are fortunate with Forests to have the broad scientific backing and publishing experience of MDPI, an international organization located in Basel, Switzerland, that has been publishing peer-reviewed, full Open Access scholarly journals since 1996.

Finally, the Editorial Board views Forests as an innovative publication for forestry-related research. Our collective goal is to have Forests be recognized as one of the foremost publication outlets for high quality, leading edge research in this broad and diverse field. This goal, of course, will be dependent 
upon the readership and potential authors. We, therefore, invite you to submit your original research articles, review papers, notes and suggestions for Special Issues to Forests and, in doing so, share your important research findings within the global forestry community.

\section{Appendix}

I would like to thank all 632 reviewers:

\begin{tabular}{|c|c|c|}
\hline Paddy Abbot & Raili Hokajärvi & Trent Penman \\
\hline Robert C. Abt & Jarmo K. Holopainen & David Pepper \\
\hline Alexis Achim & Peter Homann & George L. W. Perry \\
\hline Wouter Achten & Elaine Hooper & Matthew Peters \\
\hline Hector Guy Adégbidi & Coeli Hoover & Davide Pettenella \\
\hline Razi Ahmed & David Howat & Jacob Phelps \\
\hline Johan Ahnström & Cho-ying Huang & Marie-Gabrielle Piketty \\
\hline Tetsuya Aikoh & Shengli Huang & Neal Pilger \\
\hline Jennifer Alix-Garcia & Robert Hubbard & Brad Pinno \\
\hline Craig R. Allen & Andrew Thomas Hudak & Gianluca Piovesan \\
\hline Daniel M. Alongi & Jiri Hulcr & Benno Pokorny \\
\hline Henry Amerson & Shoana Humphries & Philip Polglase \\
\hline Hans-Erik Andersen & Len M. Hunt & David Pothier \\
\hline Krister Andersson & Lindsay B. Hutley & Matt Powers \\
\hline Tommaso Anfodillo & William Hyde & S. Andrew Predmore \\
\hline Arild Angelsen & Jyrki Hytonen & Evan L. Preisser \\
\hline Peter Annighöfer & Juha Нyуррӓ & Caroline Preston \\
\hline Altaf Arain & J. Bosco Imbert & Hans Pretzsch \\
\hline Dolors Armenteras-Pascual & Masahiro Inagaki & Hari Priyadi \\
\hline Jaume Arnó & John Innes & Timo Pukkala \\
\hline María L. Arosa & Fikret Isik & Francis E. Putz \\
\hline Fred O. Asiegbu & Taiichi Ito & Audrey Quentin \\
\hline Michael J. Aspinwall & Steven Jack & John Raison \\
\hline Stibniati Atmadja & William R. Jacobi & Serge Rambal \\
\hline Martin K.-F. Bader & Douglass F. Jacobs & Jon Ranson \\
\hline Jan R. Bannister & Patrick James & Livia Rasche \\
\hline Gonzalo G. Barberá & Schuler Jamie & Catherine Ravenscroft \\
\hline Grenville Barnes & Robert Jandl & Duncan Ray \\
\hline Luke Barry & Anna M. Jensen & Carolyn Raymond \\
\hline Jonathan Bauer & Jennifer Jensen & Gerald E. Rehfeldt \\
\hline Jenise Bauman & Tord Johansson & Mary Reid \\
\hline Karen M. Bayne & Dale W. Johnson & Marcel Rejmanek \\
\hline Chris Beadle & Dustin Johnson & James S. Rentch \\
\hline Daniel Bebber & Kristofer Johnson & Stephen E. Reutebuch \\
\hline Pablo I. Becerra & Dana M. Johnsonn & José M. Rey Benayas \\
\hline
\end{tabular}




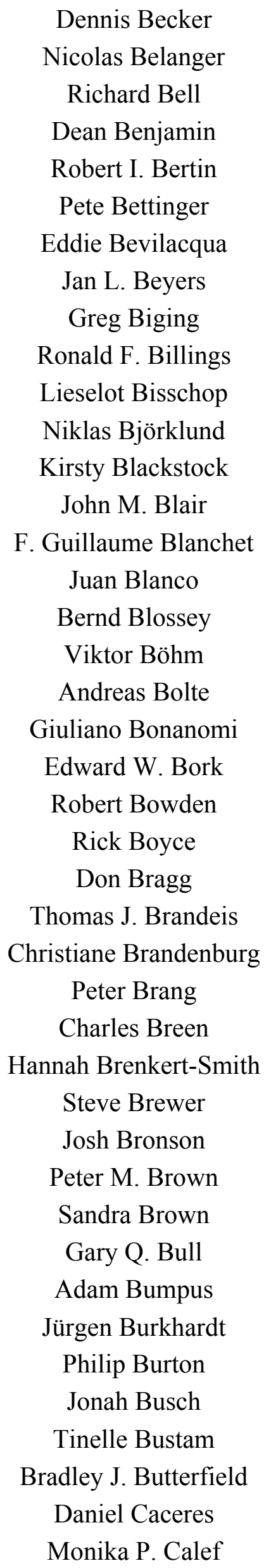

\begin{tabular}{|c|}
\hline Mariann Johnston \\
\hline Bengt-gunnar Jonsson \\
\hline Anna Maria Jönsson \\
\hline Chuleui Jung \\
\hline Virpi Junttila \\
\hline David Kaczan \\
\hline Jeffrey Kane \\
\hline Jeffrey M. Kane \\
\hline Mike Kane \\
\hline Annika Susanna Kangas \\
\hline Ville Kankare \\
\hline Peter J. Kanowski \\
\hline Elizabeth J. Stamm Katovich \\
\hline Rodney Keenan \\
\hline Michael M. Keller \\
\hline Werner Kerz \\
\hline Christopher Keyes \\
\hline Azad Henareh Khalyani \\
\hline Partap Khanna \\
\hline Anthony Kiem \\
\hline Georg Kindermann \\
\hline Mengistie Kindu \\
\hline Nalin Kishor \\
\hline Andres Kiviste \\
\hline Christoph Kleinn \\
\hline Heather Klemick \\
\hline Christie Ann Klimas \\
\hline Robert Klinger \\
\hline Eric Knapp \\
\hline Paul Knapp \\
\hline Miroslav Kolarík \\
\hline Katri Kostiainen \\
\hline Jacek Kozak \\
\hline Marek Krasowski \\
\hline Markus Kröger \\
\hline Eric Kruger \\
\hline Linda Kruger \\
\hline Christoph Kueffer \\
\hline Sunil Kumar \\
\hline John Kush \\
\hline Osmo Kuusi \\
\hline Anne C. Lang \\
\hline
\end{tabular}

Yuri Ribakov

Bryce A. Richardson

Martin Ricker

Philippe Rigault

David Rivest

Daniel Robison

John S. Roden

Rolando Rodríguez

Roque Rodríguez-Soalleiro

Raúl Romero-Calcerrada

Petri Rönnholm

Amy. Y. Rossman

Philippe Rozenberg

Thomas K. Rudel

Paloma Ruiz-Benito

Matthew B. Russell

Ervan Rutishauser

Rose-marie Rytter

Tara E. Sackett

Cuauhtemoc Saenz Romero

Jirky Salmi

Berit Samils

Lisa J. Samuelson

Chris Sandbrook

Maurizio Santoro

Nophea Sasaki

Tamara Heartsill Scalley

John Schelhas

Robert M. Scheller

Steve Schill

Jacqueline Schirmer

Robert Schmidt

Silvio Schuler

Olaf Schwab

Andrew Scott

Klaus Seeland

Rupert Seidl

Paolo Semenzato

Robert Seymour

Ram P. Sharma

William P. Shepherd

Rosemary Sherriff 


\begin{tabular}{|c|c|}
\hline Phil Cannon & Elizabeth Lapoint \\
\hline Angus Carnegie & Guy R. Larocque \\
\hline David Carr & Alejandra Larrazabal \\
\hline Jose Antonio Carreira & Martin Lascoux \\
\hline João M.B. Carreiras & Daniel Laughlin \\
\hline Marco Carrer & Silke Lautner \\
\hline Robert Carter & Martin Lavoie \\
\hline Gaetano Castro & Jessica Leahy \\
\hline Andrea Cattaneo & Luc LeBel \\
\hline Csaba Centeri & Woo-Kyun Lee \\
\hline Sun Joseph Chang & Zakiya Leggett \\
\hline Gang Chen & Tarja Lehto \\
\hline Pei-ti Chen & Stacey A. Leicht-Young \\
\hline Paolo Cherubini & Reik Leiterer \\
\hline Jyh-Min Chiang & Laura P. Leltes \\
\hline Simon Choge & Jingjing Liang \\
\hline Chris Cieszewski & Andrew Liebhold \\
\hline Miguel Cifuentes & Yi Lin \\
\hline Marco Ciolli & Jenny Lindén \\
\hline Stacy Clark & Staffan Lindgren \\
\hline Raymond A. Cloyd & Marcus Lindner \\
\hline Dean Coble & Zoë Lindo \\
\hline Francis Colin & Christina M. Locke \\
\hline Luke Collins & Brian Lockhart \\
\hline Stephen J. Colombo & Edward F. Loewenstein \\
\hline Lucy Commander & Fabio Lombardi \\
\hline Joseph L. Conrad Conrad & Bill Loneragan \\
\hline John Cowie & Baastian Louman \\
\hline David Coyle & Jenny Lovell \\
\hline Wendell Cropper & Cath Lovelock \\
\hline Frederick Cubbage & Pengxin Lu \\
\hline Harry Cullinan & Ruben Lubowski \\
\hline Bob Curtis & Ariel Lugo \\
\hline Peter Curtis & Tom Lynch \\
\hline Mark Cutler & Meghan Graham MacLean \\
\hline Anthony W. D'Amato & Alexander J. Macpherson \\
\hline Shree Dangal & Annikki Makela \\
\hline John Day & Harri Mäkinen \\
\hline Will de Jong & Marja Maljanen \\
\hline David B. de Vallance & Anna Malmstrom \\
\hline Gaby Deckmyn & Pille Mänd \\
\hline Juan Declet-Barreto & Udo Mantau \\
\hline
\end{tabular}

Tadashi Shimizu

Richard Sikkema

Martin Simard

Javier A. Simonetti

Tommaso Sitzia

Ken Skog

Hans Skov-Petersen

Margaret Skutsch

Margareth Skutsch

Bill Slee

Bernard Slippers

Ronald J. Smernik

Tat Smith

Barry Solomon

Fran Søndergaard Jensen

Bo Song

Denis Sonwa

Peter Soule

John Spence

Raffaele Spinelli

David Spittlehouse

Golo Stadelmann

Ken Stadt

Göran Ståhl

Christina Staudhammer

Robert Van Steenwyk

Taylor Stein

Martyna Stelmaszczuk-Gorska

Anothony Stocks

Benoît St-Onge

Niels Strange

Charlotte Streck

Kathrin Streit

Juliet C. Stromberg

Wayne Strong

Inge Stupak

Juan C. Suárez

Benjamin Sullivan

Tom Sullivan

Guoqing Sun

Andres I. Susaeta

Brent Swallow 
Luiz Eduardo V. Del Bem

Tom Deluca

Sergio De-Miguel

Jinyang Deng

R. Justin DeRose

Eric Dewolf

Narayan Dhital

Phillip Dougherty

Vladimir Douhovnikoff

Paul L. Drake

Brian Drayton

Rein Drenkhan

Mark J. Ducey

Isabelle Duchesne

Peter Duinker

Michael Dutschke

Klaus Edenhoffer

Dominic Elson

Leif Eriksson

Timothy J. Fahey

John Fellers

Jean-baptiste Feret

Paulo Fernandes

Ivan Fernandez

Leena Finér

Reiner Finkeldey

David Flaspohler

Teresa Fidalgo Fonseca

Irwin N. Forseth

Brian Forstler

Julien Fortier

Thomas Fox

Art Fredeen

Joy Fritschle

Lucía Galiano

Niko Galiatsatos

Glenn Galloway

Gustavo A. Garcia-Lopez

J. Garcia-Pausas

Maria João Gaspar

Chris Gentry

René H. Germain
Stefano Manzoni

Danielle Marceau

Dave Marcouiller

Robert E. Marra

Adrian Martin

Tim Martin

Grit Martinez

Cristina Martínez-Garza

Jordi Martínez-Vilalta

Axel Marx

Bill Mason

Tara Joy Massad

Eskil Mattsson

Steven Lee Matzner

Aixchel Maya-Martínez

Brian C. McCarthy

Dawn Mccarthy

George Mccaskill

Lachie Mccaw

T. Eric Mcconnell

M. Luke McCormack

Constance McDermott

Cynthia Mcdougall

Chris Mcelhinny

Ryan Mcewan

Bonnie McFarlane

Robert Mcgaughey

Chris Mcglone

Anne C.S. McIntosh

I.r. Mcivor

Craig Mckinley

Ronald E. McRoberts

Steve Meadows

Arjan J. Meddens

Fan-rui Meng

Michael Messina

Marek Metslaid

Patrick Meyfroidt

Christine N. Meynard

Richard Michalet

Jessica Miesel

Jari Miina
Anu Swatantran

Jennifer J. Swenson

Ruth Swetnam

Mary Anne Sword Sayer

Angela Taboada

Ayco J. M. Tack

David Takacs

Bruce Talbot

Sara R. Tanis

Frank W. Telewski

Christian Temperli

Naresh Thevathasan

Evelyne Thiffault

Magnus Thor

Mulualem Tigabu

Jenna Tilt

Anne Toppinen

Sylvie Tremblay

Carl C. Trettin

Takahiro Tsuge

Arvo Tullus

Sakari Tuominen

Mary Tyrrell

Liisa Tyrväinen

Michael Ulyshen

Raúl Abel Vaca

Annukka Vainio

Rubén Valbuena

Harry T. Valentine

Erik Valinger

V. Ramón Vallejo

Peter van der Meer

Paul van Deusen

Thi van Tran

G. Peter van Walsum

Lukas van Zwieten

Jari Vauhkonen

Bruno Verbist

Louis Verchot

Brian Via

Jason Vogel

Jim Vogelmann 


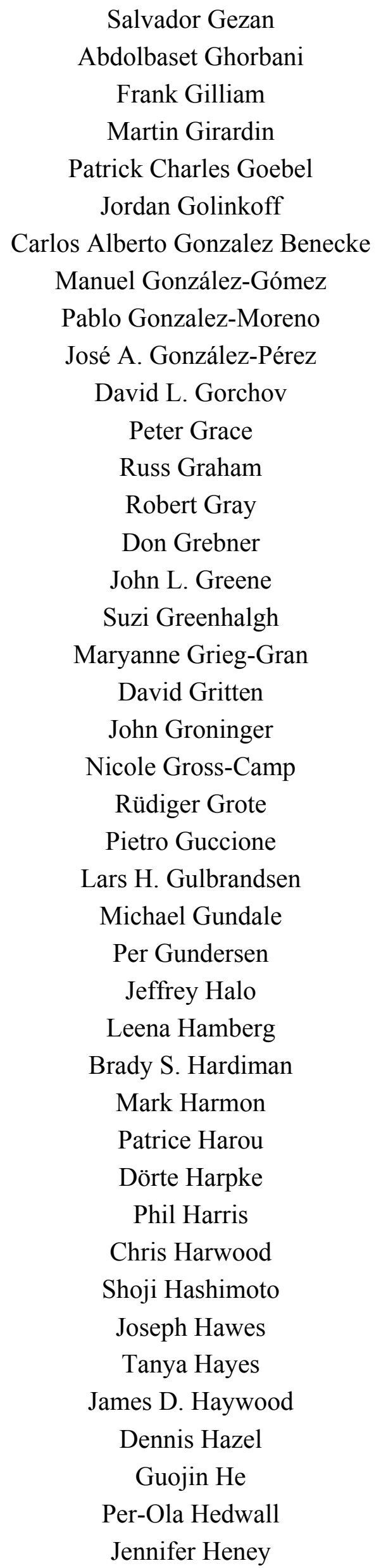

Bradley Miller

Carol Miller

Patrick Minogue

Jaconette Mirck

Iliana Monterroso

John Moore

David Moorhead

Martin Moroni

Dave Morris

Lawrence Morris

Stuart A. Moss

Hassan Muhamed

Jörg Müller

Robert Müller

Brenda L. Murphy

Wayne Myers

Gert-jan Nabuurs

Masahiko Nakagawa

Iben Nathan

Carla Nati

Jose Návar

Lucas E. Nave

Daniel Neary

Prakash Nepal

Seppo Neuvonen

World Nieh

Maarten Nieuwenhuis

Gordon Nigh

Craig R. Nitschke

Tanaka Nobuhiko

Tomas Nordfjell

Thomas Nord-Larsen

Daivd Norton

Peter Nosko

Izaya Numata

Victoria Nuzzo

Kazuharu Ogawa

Andras Bela Olah

Julianne O'reilly-Wapstra

Hans Ole Orka

Robert O'sullivan

Bernt-Håvard Øyen
Sonja Vospernik

Peter Waddell

Matthew J. Waghorn

Alaka Wali

John C. F. Walker

Deborah Ann Waller

David Walter

Bradley B. Walters

Changlu Wang

Hsiao-hsuan Wang

Kristen Waring

Ian Warkentin

Tsunehiro Watanabe

James Watling

Martin Wattenbach

Danielle A. Way

Pascale Weber

Christopher R. Webster

Aaron Weiskittel

Philip W. West

Chris Weston

Piotr Wezyk

Ross W. Whetten

Alan White

Eric White

Paul Whitehead

Whitney Whitney

Rodney Will

Roger Williams

Duncan Wilson

Sandra Wochele

Keith Woeste

Paul Woodcock

Alex Woods

Wei Wu

Tanya Wyatt

Vikram Yadama

Fukuju Yamamoto

Jeng-tze Yang

Zhiqiang Yang

Tian Yao

Runsheng Yin 


$\begin{array}{ccc}\text { David Hewitt } & \text { Pablo Pacheco } & \text { Giuliana Zanchi } \\ \text { Gordon Hickey } & \text { Brian Palik } & \text { Yaoqi Zhang } \\ \text { Barbara Hock } & \text { Sebastián Palmas } & \text { Guang Zheng } \\ \text { William Hockaday } & \text { Riikka Paloniemi } & \text { Dimitris Zianis } \\ \text { Kent Hoekman } & \text { Geoffrey Parker } & \text { Pieter Zuidema } \\ \text { Anja A. Hoffmann } & \text { William C. Parker } & \text { Natalie Zurbriggen } \\ \text { Nick Hogarth } & \text { John Parton } & \text { Janusz Zwiazek } \\ \text { Tore Högnäs } & \text { Jari Parviainen } & \end{array}$

(C) 2014 by the authors; licensee MDPI, Basel, Switzerland. This article is an open access article distributed under the terms and conditions of the Creative Commons Attribution license (http://creativecommons.org/licenses/by/3.0/). 